\title{
Gradual Ordering in Red Abalone Nacre
}

\author{
P. U. P. A. Gilbert, ${ }^{, \dagger, \S}$ Rebecca A. Metzler, ${ }^{\dagger}$ Dong Zhou, ${ }_{\ddagger}^{\dagger}$ Andreas Scholl, ${ }^{\ddagger}$ Andrew Doran, ${ }_{+}^{\ddagger}$ Anthony Young, Martin Kunz, ${ }^{\ddagger}$ Nobumichi \\ Tamura, and Susan N. Coppersmith ${ }^{\dagger}$
}
Department of Physics, UniVersity of Wisconsin, Madison, Wisconsin 53706 and AdVanced Light Source, Lawrence Berkeley National Laboratory, Berkeley, California 94720

Received August 18, 2008; E-mail: pupa@physics.wisc.edu

\begin{abstract}
Red abalone (Haliotis rufescens) nacre is a layered composite biomineral that contains crystalline aragonite tablets confined by organic layers. Nacre is intensely studied because its biologically controlled microarchitecture gives rise to remarkable strength and toughness, but the mechanisms leading to its formation are not well understood. Here we present synchrotron spectromicroscopy experiments revealing that stacks of aragonite tablet crystals in nacre are misoriented with respect to each other. Quantitative measurements of crystal orientation, tablet size, and tablet stacking direction show that orientational ordering occurs not abruptly but gradually over a distance of $50 \mu \mathrm{m}$. Several lines of evidence indicate that different crystal orientations imply different tablet growth rates during nacre formation. A theoretical model based on kinetic and gradual selection of the fastest growth rates produces results in qualitative and quantitative agreement with the experimental data and therefore demonstrates that ordering in nacre is a result of crystal growth kinetics and competition either in addition or to the exclusion of templation by acidic proteins as previously assumed. As in other natural evolving kinetic systems, selection of the fastest-growing stacks of tablets occurs gradually in space and time. These results suggest that the self-ordering of the mineral phase, which may occur completely independently of biological or organic-molecule control, is fundamental in nacre formation.
\end{abstract}

\section{Introduction}

Nacre, or mother-of-pearl, is widely studied because of its self-assembled, efficient, and accurately ordered architecture, toughness, and fascinating and poorly understood formation mechanisms. Haliotis rufescens (red abalone) and all other gastropods form columnar nacre: a composite of layered $400 \mathrm{~nm}$ thick aragonite crystalline tablets oriented with their [001] crystal axes spread around the normal to the layer plane $^{6-8}$ and stacked irregularly and $30 \mathrm{~nm}$ thick organic matrix sheets mostly composed of -chitin. Aragonite, a hard but brittle orthorhombic CaCO3 polymorph, accounts for 95\% of nacre's mass, yet nacre is 3000 -fold tougher than aragonite. No synthetic composites outperform their components by such large factors. It is therefore of great interest to understand nacre formation.

We present here X-ray photoelectron emission spectromicroscopy (X-PEEM) data on H. rufescens shells that yield unprecedented detailed and quantitative information about the location, size, and orientation of individual tablets with submicrometer spatial resolution, a large field of view including hundreds of tablets, straightforward sample preparation, and negligible radiation damage. The data exhibit a systematic spatial variation, indicating that the aragonite tablets in nacre order gradually, and are consistent with a theoretical model in which aragonite crystal layers are nucleated sequentially in the presence of confining matrix sheets and grow epitaxially on aragonite crystals in layers below. All tablets compete for space, and those with $c$ axes oriented normal to the layers prevail over misoriented tablets.

Illuminating polycrystalline samples with polarized soft X-rays generates a spectroscopic effect known as X-ray linear dichroism ${ }^{12,13}$ in X-ray absorption near-edge structure (XANES)

$\dagger$ University of Wisconsin.

† Lawrence Berkeley National Laboratory. ${ }^{\S}$ Previously publishing as Gelsomina De Stasio.

(1) Weiner, S.; Lowenstam, H. A.; Hood, L. Proc. Natl. Acad. Sci. U.S.A. 1976, 73, 2541-2545.

(2) Addadi, L.; Weiner, S. Proc. Natl. Acad. Sci. U.S.A. 1985, 82, 4110-

(3) Nassif, N.; Pinna, N.; Gehrke, N.; Antonietti, M.; Jager, C.; Colfen, . Proc. Natl. Acad. Sci. U.S.A. 2005, 102, 12653-12655.

(4) Belcher, A. M.; Wu, X. H.; Christensen, R. J.; Hansma, P. K.; Stucky,

G. D.; Morse, D. E. Nature 1996, 381, 56-58.

(5) Metzler, R. A.; Abrecht, M.; Olabisi, R. M.; Ariosa, D.; Johnson, C. J.; Frazer, B. H.; Coppersmith, S. N.; Gilbert, P. U. P. A. Phys. ReV. Lett. 2007, 98, 268102.

(6) Metzler, R. A.; Zhou, D.; Abrecht, M.; Chiou, J. W.; Guo, J. H.; Ariosa, D.; Coppersmith, S. N.; Gilbert, P. U. P. A. Phys. ReV.B 2008, 77, 064110.

(7) Chateignera, D.; Hedegaard, C.; Wenk, H.-R. J. Struct. Geol. 2000, 22, 1723-1735.

(8) Hedegaard, C.; Wenk, H.-R. J. Molluscan Stud. 1998, 64, 133-136.

(9) Levi-Kalisman, Y.; Falini, G.; Addadi, L.; Weiner, S. J. Struct. Biol. 2001, 135, 8-17.

(10) Currey, J. D. Proc. R. Soc. London B 1977, 196, 443-463.

(11) Gao, H.; Ji, B.; Jager, I. L.; Arzt, E.; Fratzl, P. Proc. Natl. Acad. Sci.

U.S.A. 2003, 100, 5597-5600.

(12) Stohr, J.; Baberschke, K.; Jaeger, R.; Treichler, R.; Brennan, S. Phys. ReV. Lett. 1981, 47, 381-384.

(13) Stohr, J.; Samant, M. G.; Luning, J.; Callegari, A. C.; Chaudhari, P.; Doyle, J. P.; Lacey, J. A.; Lien, S. A.; Purushothaman, S.; Speidell, J. L. Science 2001, 292, 2299-2302. 
A

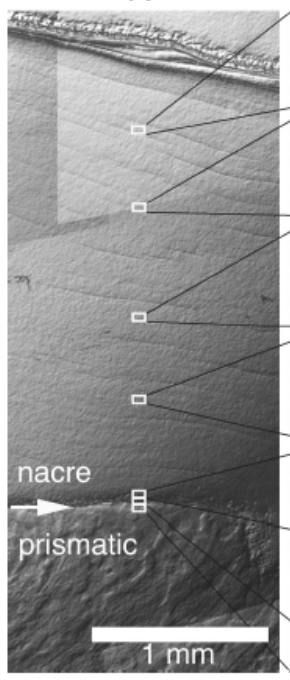

B

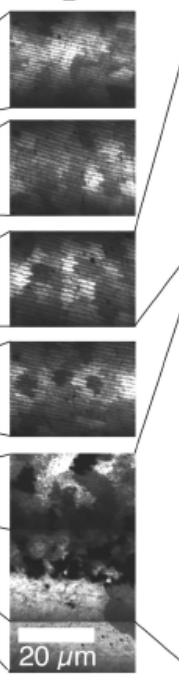

C

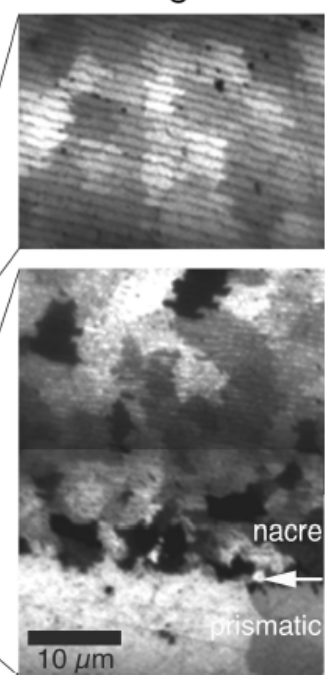

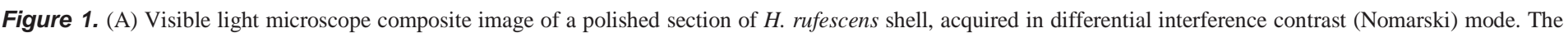

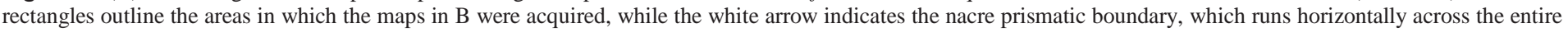

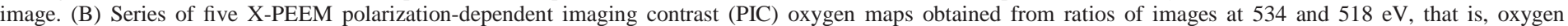

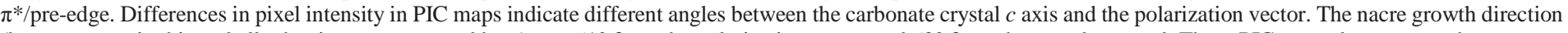

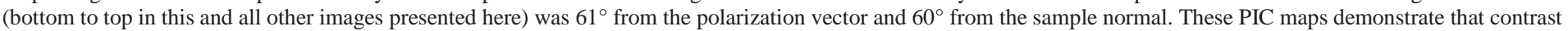

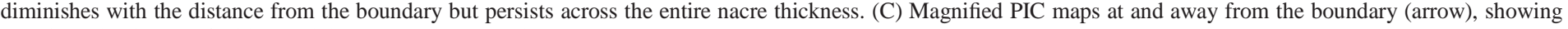
co-oriented columns of tablets and the contrast between them.

spectra. ${ }_{13}^{14}$ This effect is widely studied in manmade liquid crystals, chemisorbed and magnetic materials, and occasionally in geologic minerals ${ }_{5}^{17,18}$ and was recently discovered in nacre. When XANES spectroscopy is combined with X-PEEM imaging, the linear dichroism generates imaging contrast between differently oriented adjacent microcrystals, denoted polarization-dependent imaging contrast (PIC). Because of aragonite's symmetry in the $a b$ plane, the contrast arises from differences in the crystals' $c$ axes orientation. ${ }^{6,14,19}$ In this work we analyzed 11 samples from four different $H$. rufescens shells, approximately $10 \mathrm{~cm}$ long, with two X-PEEM instruments: the Spectromicroscope for photoelectron imaging of nanostructures with X-rays (SPHINX) at the Synchrotron Radiation Center (SRC), and PEEM-3 at the Advanced Light Source (ALS). The shells were cut, embedded, polished, and Pt coated as described in ref 22.

\section{Results}

Figure 1 shows oxygen PIC maps acquired with SPHINX at and away from the prismatic boundary. In Figure 1 adjacent

(14) Stohr, J. NEXAFS Spectroscopy; Springer-Verlag: Berlin, 1992.

(15) Roth, C.; Hillebrecht, F. U.; Rose, H. B.; Kisker, E. Phys. ReV. Lett. 1993, 70, 3479-3482.

(16) Stohr, J.; Scholl, A.; Regan, T. J.; Anders, S.; Luning, J.; Scheinfein,

M. R.; Padmore, H. A.; White, R. L. Phys. ReV. Lett. 1999, 83, $1862-1865$.

(17) Templeton, D. H.; Templeton, L. K. Acta Crystallogr., Sect. A 1997, 53, 352-355.

(18) van Aken, P. A.; Lauterbach, S. Phys. Chem. Miner. 2003, $30,469-477$.

(19) Zhou, D.; Metzler, R. A.; Tyliszczak, T.; Guo, J.; Abrecht, M.; Coppersmith, S. N.; Gilbert, P. U. P. A. J. Phys. Chem. B 2008, 112, 13128-13135. tablets exhibit strikingly strong contrast, as shown by the different gray levels, due to different orientations of adjacent crystalline tablets in each nacre layer. We note that oxygen and carbon PIC maps, ratios of images acquired at the $\pi^{*}$ and $\sigma^{*}$ energies, exhibit the same gray level intensity in each pixel and thus the same contrast between adjacent crystalline tablets.

Theory predicts that the $\pi^{*}$ intensity depends only on the polar angle $\theta$ and has a $\cos ^{2} \theta$ dependence, consistent with the experimental data in Figure 2A. Others report that nacre tablets are misoriented in the $a b$ plane ${ }_{8,27,28}^{8,23-27}$ or that $c$ axes have a spread at the macroscopic scale, ${ }^{8,27,28}$ but to our knowledge our recent observations reported here and elsewhere provide the first evidence of $c$ axis orientation variations in immediately adjacent nacre tablets. The experimental data shown in Figure 2B demonstrate that the PIC contrast arises from misorientation of the $c$ axes with respect to each other and to the polarization vector, while tablet rotation around the $c$ axes, or crystal

(20) Frazer, B. H.; Girasole, M.; Wiese, L. M.; Franz, T.; De Stasio, G. Ultramicroscopy 2004, 99, 87-94.

(21) http://xraysweb.lbl.gov/peem2/webpage/Home.shtml.

(22) Gilbert, P. U. P. A.; Abrecht, M.; Frazer, B. H. In Molecular Geomicrobiology; Banfield, J. F., Cervini-Silva, J., Nealson, K. H., Eds.; MSA: Washington, D.C., 2005; Vol. 59, pp 157-185.

(23) DiMasi, E.; Sarikaya, M. J. Mater. Sci. 2004, 19, 1471-1476.

(24) Feng, Q. L.; Cui, F. Z.; Pu, G.; Wang, R. Z.; Li, H. D. Mater.

Sci. Eng., C 2000, 11, 19-25.

(25) Feng, Q. L.; Li, H. B.; Cui, F. Z.; Li, H. D.; Kim, T. N. J.

Mater. Sci. Lett. 1999, 18, 1547-1549.

(26) Hou, W. T.; Feng, Q. L. J. Cryst. Growth 2003, 258, 402-408. (27) Chateigner, D.; Hedegaard, C.; Wenk, H.-R. J. Struct. Geol. 2000, 22, 1723-1735.

(28) Checa, A. G.; Rodrı'guez-Navarro, A. B. Biomaterials 2005, 26, 1071- 1079. 

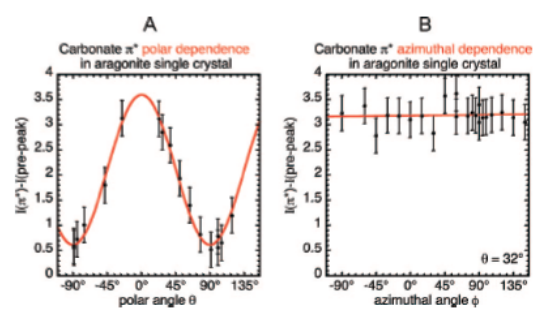

Figure 2. Angular dependence of the carbon $\pi^{*}$ peak intensity from a macroscopic single crystal of aragonite. (A) The in-plane crystal $c$ axis was rotated, with the polarization vector fixed and horizontal. The polar angle $\theta$ between polarization and $c$ axis was varied between $-90^{\circ}$ and $+120^{\circ}$. A each angle a carbon spectrum was collected, and its $\pi^{*}$ peak intensity was reported (black dots). The red curve is a fit of the experimental data $(I) m 1+$ $m 2 \cos ^{2} \theta$ with fit parameters $m 1$ ) $0.61, m 2$ ) $2.98,{ }^{2}$ ) 0.2 ). (B) With the polar angle fixed at $\theta) 32^{\circ}$, the sample was rotated in the $a b$ plane around the $c$ axis. The azimuthal angle $\varphi$ was varied, and again the $\pi^{*}$ peak intensity was reported. These data show no variation with $\varphi$, as shown by the flat distribution of data points. A linear fit of the data is shown in red $(I) m 3+$ $m 4 \varphi ; m 3$ ) $3.18 ; m 4$ ) 0.0002).

twinning, could not generate the contrast observed. While PIC images do not yield quantitative information on the angle of the $c$ axis of each tablet in nacre, differently oriented tablets can be identified.

The Haliotis rufescens shell samples were all mounted with the growth direction (perpendicular to the organic matrix sheets) vertical and in-plane on the polished sample surface and then imaged with the prismatic layer at the bottom and nacre on top.

The source of PEEM-3 at the ALS is a state-of-the-art elliptically polarizing undulator in which the polarization can be rotated $\left(0^{\circ}-90^{\circ}\right)$ to maximize the contrast. For all samples analyzed using PEEM-3, the polarization was rotated from vertical (parallel to the growth direction) to horizontal (perpendicular). Maximum contrast was obtained in all cases at $45^{\circ}$, consistent with the inflection point at $45^{\circ}$ in Figure $2 \mathrm{~A}$; around this angle small variations of angle cause large variations in the $\pi^{*}$ peak intensity, yielding large contrast between bright and dark tablets in PIC images.

Figure 3A, a carbon PIC image taken near the nacre-prismatic boundary, shows stacks of co-oriented tablets with the same gray level, consistent with the idea that mineral bridges ${ }^{30,31}$ through a single but porous organic nucleation site transmit the orientation of crystals across successive layers. We observe here that the stacks of co-oriented tablets have finite height, so it is plausible that a fraction of nucleation sites nucleate new crystal orientations.

(29) Mutvei, H. Zool. Scr. 1978, 7, 287-296.

(30) Schaffer, T. E.; Ionescu-Zanetti, C.; Proksch, R.; Fritz, M.; Walters, D. A.; Almqvist, N.; Zaremba, C. M.; Belcher, A. M.; Smith, B. L.; Stucky, G. D.; Morse, D. E.; Hansma, P. K. Chem. Mater. 1997, 9, 1731-1740.

(31) Cartwright, J. H.; Checa, A. G. J. R. Soc. Interface 2007, 4, 491-504. (32) Nudelman, F.; Gotliv, B. A.; Addadi, L.; Weiner, S. J. Struct. Biol. 2006, 153, 176-187.

(33) Song, F.; Zhang, X. H.; Bai, Y. L. J. Mater. Res. 2002, 17, 1567-1570

(34) Nakahara, H. Venus, Jap. J. Malachol. 1979, 38, 205-211.

(35) Nakahara, H.; Bevelander, G. Venus, Jap. J. Malachol. 1982, 41, 33- 46.

(36) Nakahara, H. In Biomineralization and biological metal accumulation: biological and geological perspectiVes; Westbroek, P., de Jong, E. W., Eds.; Reidel: New York, 1983; pp 225-230.

Figure 3. Experimental and simulated data on nacre ordering. (A) Carbon PIC map obtained by the ratio of $\pi^{*}$ and $\sigma^{*}$ images acquired with PEEM-3 at the ALS in a region at the nacre-prismatic boundary, separating nacre

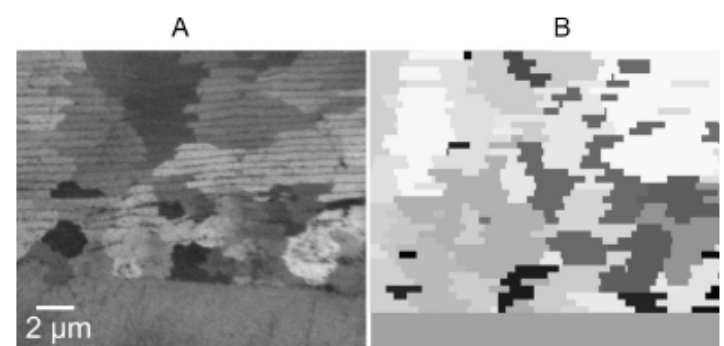

(top) and the prismatic layer (bottom), from a $H$. rufescens cross section. In this pattern of contrast stacks of tablets with the same gray level have the same crystal orientation, and the decrease in overall contrast moving away from the nacre-prismatic boundary indicates that tablet $c$ axis orientations become closer to perpendicular to the organic matrix layers. In this image the polarization vector formed an angle of approximately $45^{\circ}$ with the vertical nacre growth direction. (B) Simulation results for a model in which each layer grows to completion before the next layer is nucleated, all nucleation sites are randomly distributed with $x$ and $y$ coordinates each constrained to be within a distance $\eta$ from a nucleation site in the layer below, and each tablet adopts the orientation of the tablet below its nucleation site with probability $1-\varepsilon$. The growth rates () gray levels) of the tablets in the first layer as well as those not co-oriented with the tablet below are chosen uniformly and randomly in the range $[1-\delta / 2,1+\delta / 2]$. Parameter values are $\varepsilon$ ) $0.015, \delta$ ) 0.25 , and $\eta$ ) $1.5 \mu \mathrm{m}$. A two-dimensional vertical slice through the three-dimensional simulation is shown. Gray levels denote the growth rates of the tablets (light represents higher growth rate). The ordering moving away from the boundary, including decreasing tablet contrast, tablet size, and stacking direction, are in qualitative agreement with the experimental data.

To understand the origin of the peculiar tablet pattern revealed by PIC images, we developed a theoretical model for nacre formation that makes minimal assumptions, generated simulated nacre, and compared it to the experiments. The model focuses on nacre vertical growth and neglects radial shell growth. It makes standard assumptions well supported by experimental observations: Organic matrix sheets confine growth of the aragonite tablets ${ }^{34-36}$ and crystal growth is "poisoned" when a tablet hits the overlying organic sheet; ${ }^{37}$ There is a single nucleation site per nacre tablet; Nucleation sites are independently and randomly distributed on organic matrix sheets; Tablets in a given layer grow until they reach confluence; A tablet in a given layer is highly probable to have the same orientation as the tablet directly below its nucleation 30,31,39

site. ${ }^{30,31,39}$ The aragonite tablets in the first layer have $c$ axes that are not perfectly aligned. This assumption is supported directly by the experimental data presented here. In the simplest version of the model, appropriate for sheet nacre, growth of a given layer is completed before the next layer is nucleated. To model the columnar growth of nacre in $H$. rufescens, ${ }^{35,40}$ nucleation sites in successive layers are constrained to be within a fixed distance from the one below, set by a parameter in the model. The behavior is similar for the sheet and columnar models; the results

(37) Weiner, S.; Traub, W. In Structural aspects of recognition and assembly in biological macromolecules; Balaban, M., Sussman, J. L.; Traub, W., Yonath, A., Eds.; Balaban ISS: Rehovot and Philadelphia, 1981; pp 467-482.

(38) Rousseau, M.; Lopez, E.; Coute, A.; Mascarel, G.; Smith, D. C.; Naslain, R.; Bourrat, X. J. Struct. Biol. 2005, 149, 149-157.

(39) Song, F.; Soh, A. K.; Bai, Y. L. Biomaterials 2003, 24, 3623-3631.

(40) Fritz, M.; Belcher, A. M.; Radmacher, M.; Walters, D. A.; Hansma, P. K.; Stucky, G. D.; Morse, D. E.; Mann, S. Nature 1994, $371,49-51$. 

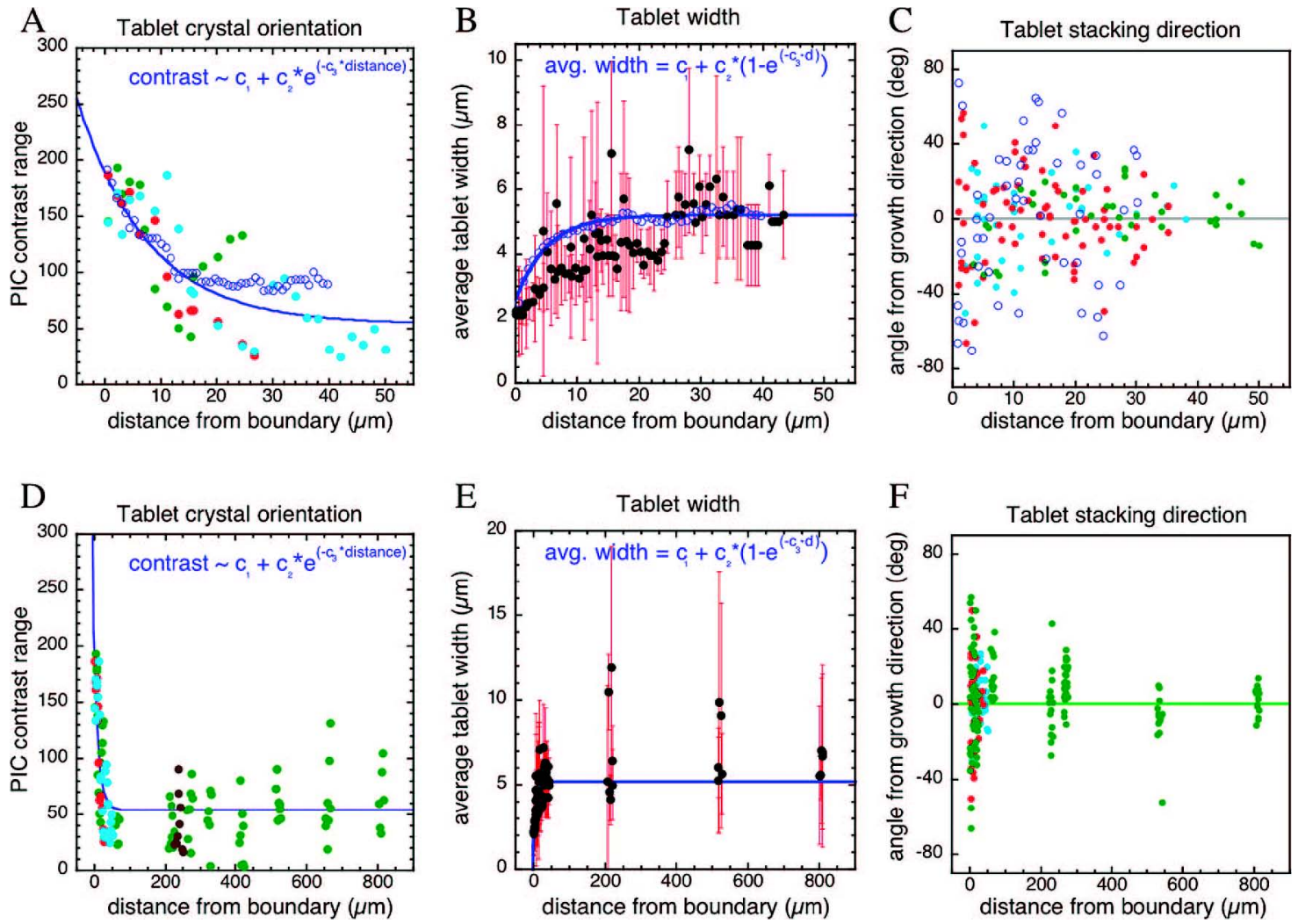

Figure 4. Quantitative measurements of tablet gray level contrast corresponding to crystal orientation, tablet width, and tablet stacking direction in nacre measured from PIC images across three shells (green, light blue, and red solid dots). All three quantities are plotted as a function of distance from the prismatic-nacre boundary, first in the short ranges $(0-50 \mu \mathrm{m})$ for all shells (A-C) and then in the long-range, up to $800 \mu \mathrm{m}$, for one shell only (D-F). The blue circles are results obtained from the theoretical model, and the blue lines are exponential fits of the model results. More details on all these data and fits are provided in Supporting Information Table S1 and Figures S9-S11. Notice the quantitative agreement between the simulation and the experimental results.

shown here are for the columnar model. In addition to the standard assumptions listed above, our model posits that there is a distribution of aragonite crystal tablet growth rates with tablets with $c$ axes oriented normal to the layers growing faster than misoriented tablets. (See Supporting Information Figure S8 for further details.) Because the sheet and columnar models behave similarly, the model is a test bed for this single hypothesis.

A simulation of the model defined by these assumptions yields the configuration shown in Figure 3B, which is strikingly similar to the experimental data in Figure 3A. The parameter $\delta$, the spread of growth rates of different crystal orientations, determines the length over which the ordering evolves, the fraction $\mathrm{E}$ of tablets that fail to adopt the crystal orientation of the tablets below their nucleation sites limits the ultimate degree of order far from the prismatic boundary, and the degree of columnarity is specified by constraining nucleation sites in successive layers to be within an in-plane distance $\eta$ from the position of the previous nucleation site. In all the figures shown in the main text $\delta$ ) 0.25 , E ) 0.015 , and $\eta$ is set to be $0.87 \mathrm{~L} / . N$, where $N$ is the number of nucleation sites in each layer and $L$ the linear dimension of the square domain simulated; thus, $\eta$ ) $1.5 \mu \mathrm{m}$.

Although PIC images do not provide numerical values for $c$ axis angles or crystal growth rates, valuable quantitative information on the evolution of the contrast, tablet size, and tablet stacking direction can be extracted, as presented in Figure

4. All these quantities show greater disorder near the prismatic-nacre boundary with order evolving over a length scale of approximately $50 \mu \mathrm{m}$. Figure 4 and Supporting Information Table S1 and Figures S9-S10 demonstrate that the model agrees quantitatively with experimental measurements of the dependence on distance from the prismatic boundary of imaging contrast and tablet widths. Figure S11 shows data for tablet stacking directions and compares to the results for the model in the layer-by-layer limit $(\eta f \infty)$. One obtains qualitative agreement between theory and experiment for the stacking directions even in this limit because of the increase in tablet widths moving away from the nacre-prismatic boundary (Figure S11).

Distributions of tablet growth rates at different distances from the prismatic boundary yielded by the model, shown in Supporting Information Figure S12, clearly show narrowing of the distribution with distance from boundary.

Figure 5 presents experimental and simulation in-plane data of a single nacre layer. Visible light microscopy (Figure 5A) shows that tablets are irregular polygons arranged in a Voronoi pattern, as previously reported by Rousseau et al. The new observation here is that the boundaries between adjacent tablets are often slightly curved. Curved tablet boundaries are characteristic of the tablet pattern obtained when there are different in-plane growth rates. Figure 5B shows a pattern with only straight edges obtained with all tablets growing out of randomly located nucleation sites at the same rate. Figure 5C shows the result of growth with a uniform distribution of growth rates between 0.85 and $1.15(\delta$ ) 0.3 ); the slightly curved tablet edges, typical of a multiplicatively weighted Voronoi construc

(41) Mu, L. Prof. Geogr. 2004, 56, 233-239. 


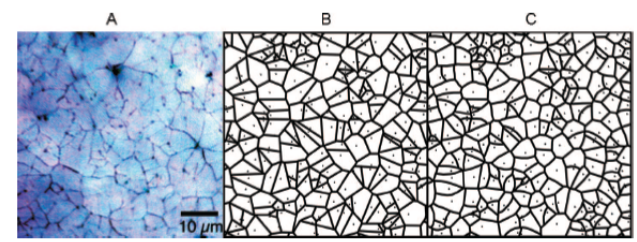

Figure 5. Experimental data and simulation results of an in-plane view of a layer of nacre tablets. (A) Visible light microscopy image obtained in differential interference contrast (Nomarski) mode from a polished nacre surface. The lines visible in this image are the boundaries between tablets. (B) Simulated layer of nacre obtained using a single isotropic growth rate for all tablets. The tablets grow radially from their nucleation sites (square dots) until they hit each other. Each point along a boundary is equidistant from the nucleation sites of two tablets, so the resulting irregular polygons form a Voronoi construction with straight lines separating any two adjacent tablets. (C) Simulated nacre layer, obtained from the same randomly distributed nucleation sites in B, but with a distribution of tablet growth rates. The resulting pattern of tablet boundaries is a multiplicatively weighted Voronoi construction, and the lines separating adjacent tablets are often curved. The growth rate spread $\delta$ ) 0.3 , similar to that used in Figure 4, yields a pattern similar to the experimental data shown in A, in which some lines are indeed curved.

tion, resemble strikingly the experimental data of Figure $5 \mathrm{~A}$. Note that this value of $\delta$ also yields good agreement with the evolution of side-view tablet widths and contrast, as shown in Figure 4.

From the Voronoi constructions in Figure 5 and those of Rousseau et al. we observe that there is one nucleation site per tablet. Nudelman et al. ${ }^{32}$ confirmed this by directly observing the nucleation sites in the organic matrix sheets, 1 or 2 at most per tablet, in Atrina rigida. These observations are in agreement with the concept of mineral bridges introduced by Scha "ffer et al., provided that the single nucleation site in the organic sheet is porous and one or more mineral bridges extend from the underlying aragonite tablet to the overlying one through this porous region of the organic sheet.

To obtain analytic insight into the model's behavior, we define $l(\gamma)$ to be the fraction of the area in layer $l$ misoriented from the normal by an angle $\gamma$. This function is a direct analog of a fitness function in population biology. Tablets with $\gamma$ ) 0 grow fastest, and therefore, their share of the area in layer $l+1$ will be greater than in layer $l$. Because the maximum is at $\gamma$ ) 0 , one expects the dependence of $l(\gamma)$ on $\gamma$ to be quadratic. Therefore

$$
\begin{aligned}
& l(\gamma) \mathrm{R} \gamma^{2} \\
& )^{+}(1-) \approx e^{1} e^{-\mathrm{R} \gamma^{2} / 2} \\
& l-1(\gamma) N l 2 N l
\end{aligned}
$$

where $\mathrm{R}$ is a numerical coefficient and $\mathrm{Nl}$ is a normalization factor that is fixed by the normalization condition

$$
\text { 1) } \left.\int^{\pi}(2 \pi \gamma)_{l}(\gamma) \mathrm{d} \gamma\right) \int_{N_{t}}^{+} \int_{l-1}^{\pi}(2 \pi \gamma) e^{-\mathrm{R} \gamma^{2} / 2}(\gamma) \mathrm{d} \gamma
$$

00

We also assume that there is a small probability $\varepsilon$ that when a tablet nucleates, instead of adopting the same $\gamma$ as the tablet below its nucleation site, it takes on a value chosen from a probability distribution $w(\gamma)$ that we will choose to be Gaussian,

$$
e^{-\gamma^{2} / 2}
$$

$w(\gamma))(() /(2 \pi))^{1 / 2}$. This misorientation process is analogous to mutation in population genetics. Therefore, the evolution is governed by the equation

$$
\begin{aligned}
& -\gamma 22_{+(1-} \underline{1}-\mathrm{R} \gamma 2 / 2 \\
& (\gamma)) \varepsilon_{(2 \pi)}^{1 / 2} e \varepsilon_{\underline{N}_{l}}^{l-1}(\gamma) e
\end{aligned}
$$

When the co-orientation of tablets in successive layers is perfect, so that $\varepsilon$ ) 0 , the solution to this equation is $l+1(\gamma) \propto 1(\gamma) e^{-l \mathrm{R} \gamma^{2} / 2}$. Therefore, when $\varepsilon$ ) 0 , for any $\mathrm{R}>0$ the width of the distribution decreases as the square root of the number of layers and becomes arbitrarily narrow as the number of layers tends to infinity.

When $\varepsilon$ is nonzero but small, so that the co-orientation between successive layers is not quite perfect, the intuitive picture of the process is that the distribution gets narrower unless a misorientation, or "mutation", occurs. The "mutations" or misorientations prevent the peak from narrowing indefinitely, so after many layers $l(\gamma)$ approaches a stationary distribution

$*(\gamma)$ that does not change as $l$ increases further. A full analysis using the techniques in ref 43 shows that the distribution $*(\gamma)$ consists of two peaks, a broad one with amplitude $\theta$ and width $-1 / 2$ and a narrow peak with amplitude $1-\theta$ and an extremely small steady-state width that is proportional to $\mathrm{e}^{-(\mathrm{CR} / \varepsilon)}$ with $C$ being a constant. Thus, the degree of ordering is limited by the reliability of the co-orientation of crystals in successive layers.

Two additional novel observations yielded by our PIC experiments are presented in Figures 6 and 7. Figure 6 shows a high-resolution PIC image of a side view of nacre tablets. Some boundaries between two adjacent tablets in the same layer are not perpendicular to the layer. The angles of tablet-to-tablet boundaries near the prismatic-nacre boundary may be as small as $25^{\circ}$ (e.g., black arrows in Figure 6A); after $50 \mu \mathrm{m}$ they are narrowly distributed around $90^{\circ}$. A possible mechanism leading to formation of such small angles, based upon the above observations indicating that the growth rates of immediately adjacent tablets may be different, is described in the caption of Figure 6B. When two neighboring tablets have significantly different growth rates, the slower-growing tablet determines the orientation of the crystal facet while the fast-growing tablet fills space. We stress that we cannot assign growth rates to gray levels; therefore, dark tablets may be faster or slower growing than light ones. The mechanism in Figure $6 \mathrm{~B}$ and $6 \mathrm{C}$, if correct, would indicate that dark tablets 1-6 are slower growing than the lighter ones.

Supporting Information Figures S14-S16 show microbeam X-ray diffraction data in agreement with the X-PEEM observations: co-oriented tablets appear as clusters of aragonite reflections rather than single spots in Laue diffraction; the $c$ axes of immediately adjacent tablets are misoriented with respect to each other, and the spread of $c$ axis orientations is $\sim 24^{\circ}$ near the prismatic-nacre boundary, gradually decaying to $\sim 6^{\circ}$ at $35 \mu \mathrm{m}$ 


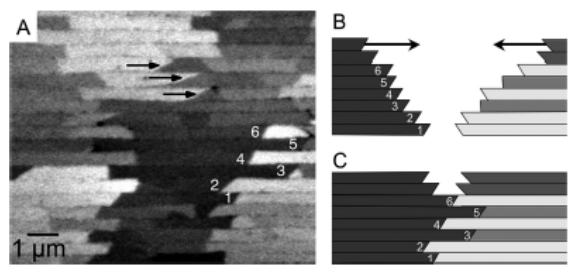

Figure 6. (A) High-resolution carbon PIC map of $H$. rufescens nacre near the nacre-prismatic boundary, showing that within a single nacre layer tablet-to-tablet boundaries are not perpendicular to the layers. Furthermore, co-oriented tablets in the same stack often exhibit similar angles in tabletto-tablet boundaries. This can be seen most strikingly in the six tablets indicated by numbers 1-6. Other areas also show a similar effect with less accurate alignment, e.g., the boundaries indicated by black arrows. This map was also acquired on PEEM- 3 at the ALS, by the ratio of $\pi * / \sigma^{*}$ images, with the polarization vector at $\sim 70^{\circ}$ from the nacre growth direction. (B) Schematic representation of tablet growth during nacre formation, assuming (arbitrarily) that the dark/light gray level corresponds to the slow/fast growth rate. The tablets on the left grow slowly toward the right, while those on the right grow faster and toward the left. The crystal facets of the dark tablets are oriented as those in tablets 1-6 in A. Light gray tablets are oriented at other arbitrary angles. (C) Snapshot of the same tablets in B at a later point in time. The slow-growing tablets have advanced little, while the fast-growing ones have advanced more. When two tablets join each other, the slow-growing tablet (e.g., tablet 1) is almost immobile and maintains its facet while the fast-growing tablet fills the remaining space, independent of its facet orientation preference. The orientation of the tabletto-tablet boundary in fully formed nacre, therefore, is only determined by the slow-growing tablets and their preferred crystal facet orientation. Slow-growing tablet 2 had a tablet-to-tablet boundary orientation similar to tablet

1. However, since it started growing later it also arrived later, so its boundary is left of 1 . Tablet 3 protrudes more to the right because it met on the right side with a tablet growing at a different rate (different gray level in A), which was presumably slower; thus, 3 overgrew 1 and 2. Tablet 4 stopped approximately where 1 and 2 did, consistent with similar gray levels on the joining right tablets. Tablet 5 overgrows 4 again, similarly to 3 , and finally, 6 follows the trend of 1,2 , and 4. Observation of nonperpendicular tablet-to-tablet boundaries as well as overgrowth of certain tablets (e.g., 3 and 5) is less frequent away from the prismatic-nacre boundary, consistent with all other data presented here that the growth rates become more homogeneous moving away from the prismatic boundary.

from the boundary. These data $\underset{8,28}{\operatorname{are}}$ in quantitative agreement with macroscopic texture analysis of nacre.

Figure 7 presents a new observation found in dozens of other PIC images acquired at the nacre-prismatic boundary of different $H$. rufescens shells: the transition from prismatic calcite to aragonite does not coincide with the onset of layered nacre formation. The first $\sim 3 \mu \mathrm{m}$ from the calcite prisms are not nacre but spherulitic aragonite crystals exhibiting great disorder in their orientation and sizes ranging between 0.5 and $2 \mu \mathrm{m}$. These crystals were identified as aragonite because their Ca L-edge spectra are typical of aragonite. $^{6,22,44}$ Calcite has very sharp and intense peaks in the crystal field region, and thus, it can be mapped as in Figure 7C. This map shows that the transition from calcite to aragonite is abrupt and that the separating line is not straight but irregularly shaped. Aragonite polymorph selection, therefore, occurs first followed by deposition of nacre organic matrix sheets and growth of the familiar layered nacre structure. Although nacre is invariably aragonitic, the prismatic layer of other mollusks, e.g., nautilus, is formed by spherulitic aragonite. This spherulitic aragonite at the prismatic-nacre boundary has not been reported previously for Halitos rufescens. Interestingly, most of the aragonite spherulites in this transition zone become layered nacre without changing their crystal orientation, as can been seen in Figure 7A.

On the basis of the new observation that polymorph selection and layering are independent phenomena, we infer that the acidic proteins that enact polymorph selection must start working first, before organic matrix sheets are formed, and continue to work during all of the subsequent nacre growth.

\section{Discussion}

The simulations of Figures $3 \mathrm{~B}$ and $5 \mathrm{C}$ and the experimental data in Figures $5 \mathrm{~A}$ and $6 \mathrm{~A}$ give strong and independent indications that aragonite tablets grow at different rates in nacre. Different in-plane growth rates are evident because tablet-totablet boundaries are often curved, while in cross-section the different growth rates manifest as tablet-to-tablet boundaries at angles much smaller than $90^{\circ}$. Different growth rates for different tablets in nacre were first suggested by Wise and Nakahara based on scanning electron microscope images of the indented conical morphology of stacks of tablets at the nacre growth front in gastropod shells.

An independent line of evidence for different growth rates is provided by the agreement of the model results with the experimental data presented here. One of the assumptions of the model was in fact the existence of different growth rates. The model, however, goes further and demonstrates that selection of the fastest-growing tablets is consistent with detailed measurements of tablet patterns. Although PIC images cannot provide directly the variability of either crystal orientation or growth rate, comparison to the model indicates that the spread in growth rates is on the order of $25 \%$.

PIC imaging reveals that stacks of co-oriented (equal growth rate) tablets start and end abruptly. This is consistent with a small probability of nucleating a different crystal orientation of $\sim 1.5 \%$.

Our results provide strong evidence that the growth rates of aragonite tablets depend on crystal orientation, but the relationship between tablet growth rates and crystal growth rates remains to be elucidated. Geologic aragonite grows 10 times faster along the $c$ axis than along the $a$ axis ${ }^{47}$ and may also do so in nacre, ${ }^{48}$ but either faster or slower growth rate along the [001] direction can yield tablet growth rates that are maximized when the tablets are perpendicular to the layers, depending on how tablet nucleation and growth is biologically regulated. A possible explanation for the gradual alignment of the $c$ axis along the layer normal is as follows. Since the organically distinct nucleation sites are pre-existing, the sooner a tablet reaches the organic sheet above, the more likely it is to hit the nucleation site and thus propagate its orientation before another tablet does so. In this "competition for space" hypothesis having the fast-growing direction, the $c$ axis, aligned with the normal ensures

(42) Turelli, M. Theor. Popul. Biol. 1984, 25, 138-193.

(43) Coppersmith, S. N.; Blank, R. D.; Kadanoff, L. P. J. Stat. Phys. 1999, 97, 429-457.

(44) Benzerara, K.; Menguy, N.; Lopez-Garcia, P.; Yoon, T.-H.;

Kazmierczak, J.; Tyliszczak, T.; Guyot, F.; Brown, G. E., Jr. Proc. Natl. Acad. Sci. U.S.A. 2006, 103, 9440-9445.

(45) Wise, S. W., Jr. Science 1970, 167, 1486-1488.

(46) Wise, S. W. Ecoglae Geol. HelV. 1970, 63, 775-797.

(47) Reeder, R. J. Carbonates: Mineralogy and Chemistry; Mineralogical Society of America: Washington, D.C., 1983; Vol. 11.

(48) Lin, A.; Meyers, M. A. Mater. Sci. Eng., A 2005, 390, 27-41.

(49) Grigor'ev, D. P. Ontogeny of Minerals; Israel Program for Scientific Translations: Jerusalem, 1965.

(50) Ubukata, T. Paleontology 1994, 37, 241-261.

(51) Checa, A. G.; Okamoto, T.; Ramy'rez, J. Proc. R. Soc. B 2006, 273, 1329-1337. 
A

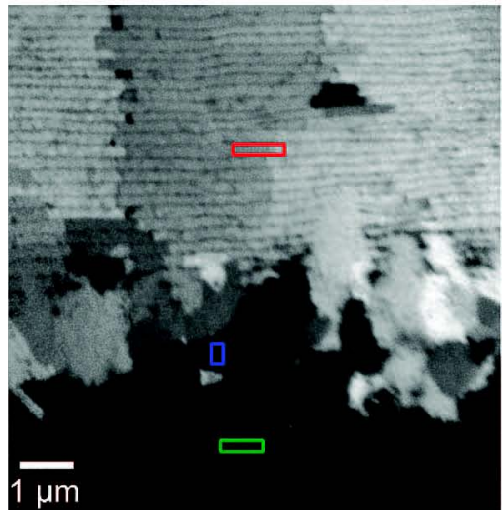

B

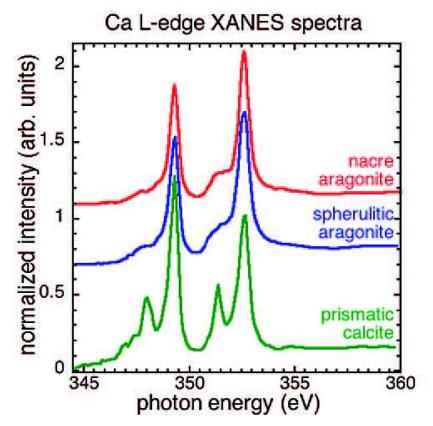

C

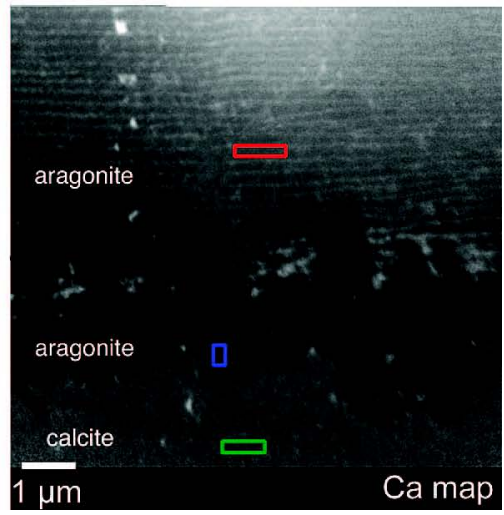

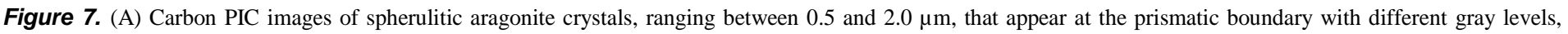

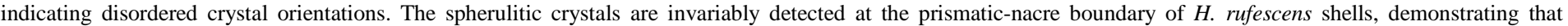

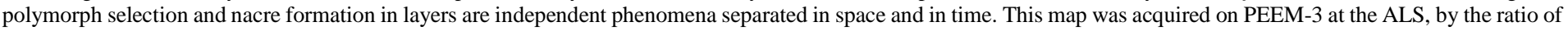

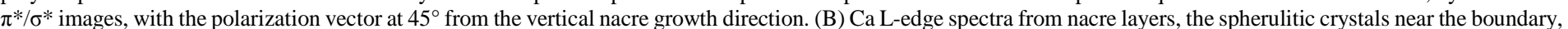

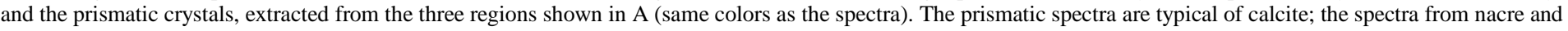

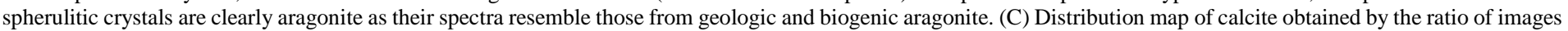

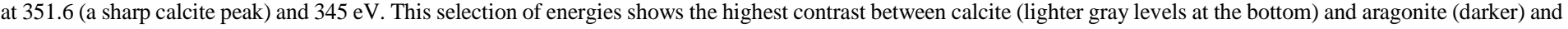
the boundary between the two polymorphs. More images of this kind are presented in Supporting Information Figure S13.

that the nucleation site is reached as soon as possible. This may explain why aligned tablets gradually prevail. This hypothesis is consistent with similar "competition for space" models in minerals and biominerals, successfully applied to sheet nacre in bivalves, in which the fast-growing direction (the $b$ axis grows 1.6 times faster than the $a$ axis in aragonite) aligns with the radial shell growth direction, and it does so gradually, ${ }_{28,51}$ as we report here for columnar nacre and the $c$ axis alignment. This similarity corroborates the present results and their interpretation with a kinetic model, which can be seen as a "competition" model. Our model, however, is more general as it also takes into account nucleation of new orientations and quantitatively explains not only the gradual crystal orientation but also the increase in tablet size.

Moreover, nacre crystals may not grow one ion at a time as in abiotic aqueous solutions. Addadi et al. and Nudelman et al. provide some evidence that sheet nacre in Atrina rigida grows from a transient amorphous calcium carbonate (ACC) phase. Having different growth rates in different directions is compatible with an ACC precursor if crystal conversion at the expense of ACC proceeds faster (or slower) along the [001] direction than along the others. Nassif et al. showed that aragonite tablets in Haliotis laeVigata nacre are surrounded by a $3-5 \mathrm{~nm}$ thick layer of stable ACC. It is conceivable that this layer is a remnant of the precursor ACC phase that failed to convert to crystalline aragonite as a result of organic molecules excluded by the rest of the crystal as proposed by the same authors, or by crystal poisoning, or by Asp-rich glycoproteins adsorbed onto the sheets.

In Figure 6B and 6C we proposed a mechanism to explain the, $90^{\circ}$ angle of tablet-to-tablet boundaries observed in Figure $6 \mathrm{~A}$. The idea that fast-growing tablets fill space independent of their crystal facet preference is consistent with the observation that the in-plane morphology of nacre tablets before confluence does not affect the final in-plane pattern of confluent tablets. In different species, in fact, nonconfluent forming nacre tablets appear as cylinders in Pinctada margaritifera, as hexagonal crystals in Atrina rigida, or as polygons in Haliotis, $\quad$ but they all converge toward final irregular polygonal patterns similar to that of
Figure $5 \mathrm{~A}$ at confluence. This demonstrates that crystals fill space until they can, independent of their crystal facet preferences. Clearly, faster-growing crystals fill space more rapidly than slow-growing ones; thus, the final tablet-to-tablet boundary is closer in morphology to the crystal facet orientation preferred by the slow-growing tablet.

The presence of spherulitic aragonite at the nacre-prismatic boundary in Haliotis rufescens has not been reported previously. Zaremba et al. report spherulitic calcite, not aragonite, preceding aragonitic nacre growth in $H$. rufescens "flat pearl". DiMasi et al. observed a jumble of randomly oriented crystals at the nacre-prismatic boundary, which is compatible with the spherulitic aragonite layer documented here at higher resolution. All other authors reported that the prismatic layer in $H$. rufescens is completely calcitic. $^{35,57}$ In bulk $H$. rufescens nacre we often observed organic growth lines followed by spherulitic aragonite (data not shown), as also observed by Su et al.

Dauphin et al. and Mutvei observed similar spherulitic aragonite at the nacre-prismatic boundary of other Haliotis species in which the prismatic layer is composed either

(52) Addadi, L.; Joester, D.; Nudelman, F.; Weiner, S. Chemistry 2006, 12, 980-7.

(53) Nudelman, F.; Che, H. H.; Goldberg, H. A.; Weiner, S.; Addadi, L. R. Soc. Chem., Faraday Discuss. 2007, 136, 9-25.

(54) Zaremba, C. M.; Belcher, A. M.; Fritz, M.; Li, Y. L.; Mann, S.; Hansma, P. K.; Morse, D. E.; Speck, J. S.; Stucky, G. D. Chem. Mater. 1996, 8, 679-690.

(55) Blank, S.; Arnoldi, M.; Khoshnavaz, S.; Treccani, L.; Kuntz, M.; Mann, K.; Grathwohl, G.; Fritz, M. J. Microsc. 2003, 212, 280-91.

(56) Gehrke, N.; Nassif, N.; Pinna, N.; Antonietti, M.; Gupta, H. S.; Co"lfen,

H. Chem. Mater. 2005, 17, 6514-6516.

(57) Dauphin, Y.; Cuif, J. P.; Mutvei, H.; Denis, A. Bull. Geol. Inst. UniV. Uppsala, N.S. 1989, 15, 7-17.

(58) Su, X.; Belcher, A. M.; Zaremba, C. M.; Morse, D. E.; Stucky, G. D.; Heuer, A. H. Chem. Mater. 2002, 14, 3106-3117.

(59) Mutvei, H.; Dauphin, Y.; Cuif, J. P. Bull. Mus. natn. Hist. nat., Paris, 4me se'r. 1985, 7, 73-91.

(60) Mutvei, H. In Origin, EVolution, and Modern Aspects of Biomineralization in Plants and Animals; Crick, R. E., Ed.; Plenum Press: New York, 1989; pp 137-151. 
exclusively by aragonite spherulites ( $H$. glabra and $H$. asinina) or a mixture of aragonite spherulites, rods, and calcitic prisms $(H$. tuberculata, $H$. lamellosa, and $H$. rotundata). ${ }^{57,59,60}$ In $H$. rufescens, however, the prismatic layer was thought to be composed exclusively of calcite prisms. ${ }^{35,57,60}$ Thus, the presence of the layer of aragonite spherulites at the nacre-prismatic boundary reported here is both unexpected and unprecedented. It is interesting to notice, however, that the spherulites reported here in $H$. rufescens appear to have an interfacial behavior, as observed in $H$. tuberculata and $H$. lamellosa: direct contact of calcitic prisms and aragonitic nacre was never observed. ${ }^{57}$ Furthermore, Dauphin et al. report gradual appearance of nacreous layers in all five aforementioned Haliotis species, exactly as observed here in $H$. rufescens. As shown in Figure 7A, the aragonite spherulites in this transition zone become layered nacre without changing their crystal orientation. A similar observation was previously reported in Nautilus by Mutvei. ${ }^{60,61}$ In Haliotis rufescens it provides further evidence for mineral bridges between nacre layers.

The spherulites at the nacre-prismatic boundary appear much more misoriented and have completely different morphology, compared to those in bulk nacre, formed on organic growth layers, but it is intriguing to connect the two observations and consider the possibility that aragonite crystals, however oriented, constitute a preferred substrate for the onset of nacre growth or regrowth, as previously observed in Nautilus ${ }^{60,61}$ and other Haliotis species. In this case, however, the absence of aragonite on "flat pearl" substrates ${ }^{54}$ is puzzling.

Acidic proteins in mollusk shells that were first reported by Gregoire ${ }^{62}$ and then found to be present in many different species were suggested to function as a template for mineralization by Weiner and Hood. In principle, it is possible that templating proteins become more aligned and therefore more effective at aligning the $c$ axes of crystals in a gradual manner, not abruptly, at the onset of nacre formation. A templation mechanism for aragonite crystal orientational ordering requires stereochemical matching of organic molecules and mineral lattice ${ }^{2}$ and is expected to determine the crystal polymorph and its orientation. Excellent data by Belcher and Falini ${ }^{4}{ }^{4}$ showed that these proteins play a key role in nucleating aragonite in vitro, thus in polymorph selection, but not in crystal orientation. To this day the only indirect evidence that orientational ordering is due to organic templation is that reported by Weiner and Traub, describing co-orientation of the -polymorph of the chitin polysaccharide crystals in the organic matrix sheet, and the overlaying aragonite crystal lattice, at least along the $a$ and $b$ axes, in several mollusk species. ${ }^{65,66}$ However, such co-orientation has never been described in Haliotis nacre, despite extensive studies on this species. Two other lines of evidence rule out templation in Haliotis: the layer of ACC surrounding each nacre tablet in Haliotis laeVigata and the completely random orientation of aragonite tablets along the $a$ and $b$ axes in the nacre of all gastropods. Checa et al. ruled out templation, or heteroepitaxy, in their model and observations of bivalve

(61) Mutvei, H. Biomineral. Res. Rep. 1972, 4, 81-86. (62) Gregoire, C.; Duchateau, G.; Florkin, M. Ann. Inst. Oceanogr. (Paris) 1955, 31,1 .

(63) Weiner, S.; Hood, L. Science 1975, 190, 987-989.

(64) Falini, G.; Albeck, S.; Weiner, S.; Addadi, L. Science 1996, 271, 67-69. nacre. ${ }^{28,51}$ The model and experiments presented here do not require templation by organic molecules but do not rule it out. We identified and clarified another component to the complex mechanism of nacre formation, that is, the fundamental role played by the mineral phase itself and its gradual ordering, which is not directly controlled biologically or organically.

\section{Conclusions}

Using state-of-the-art imaging we demonstrate that the degree of orientational order of crystalline aragonite tablets in nacre increases with distance from the prismatic boundary over a length scale of about $50 \mu \mathrm{m}$. The theoretical and experimental results presented here show that different crystal orientations in nacre tablets correspond to different growth rates. Kinetic ordering in nacre arises because oriented tablets grow faster than misoriented ones and gradually prevail in their competition for space.

A theoretical model of nacre assembly based on gradual, kinetic selection of faster growth rates and a small probability of nucleating a new orientation produces results in remarkable agreement with side-view and in-plane experimental data.

\section{Materials and Methods}

More details about the materials and methods are provided in the Supporting Information.

Samples. Haliotis rufescens shells and geologic aragonite crystals were embedded in epoxy, polished, Pt coated, and PIC imaged with one of two X-PEEM instruments: SPHINX ${ }^{20}$ at SRC and PEEM-3 ${ }^{21}$ at ALS.

The polarization-dependent imaging contrast (PIC) images were obtained by the ratio of two X-PEEM images, acquired at the $\pi^{*}$ and $\sigma^{*}$ energies of either carbon (290.3 and $302 \mathrm{eV}$ ) or oxygen (534 and $540 \mathrm{eV}$ ) since these are equivalent.

The PEEM-3 instrument differs from SPHINX in that it uses electrostatic rather than magnetic lenses, is considerably more complex and fully automated, and its source is an elliptically polarizing undulator (EPU) in which linear polarization can be rotated by $90^{\circ}$. In this work this feature was used extensively to yield the highest possible contrast for each image, which usually occurred with the polarization at $45^{\circ}$, since all samples were mounted with the growth direction oriented vertically.

Simulation Method. Each layer in the simulation was a square of dimension $400 \times 400$ arbitrary units with 600 randomly distributed nucleation sites. Nucleation sites are restricted so that the $x$ and $y$ coordinates are each within $\eta$ ) $1.5 \mu$ mofthe coordinates of a nucleation site in the previous layer. The growth rates in the initial layer are chosen uniformly at random in the interval $[1-\delta / 2,1+\delta / 2]$. In subsequent layers, for each nucleation site, with probability $1-\varepsilon$ the tablet's growth rate is the same as for the tablet directly below the nucleation site, and with probability $\varepsilon$ it is random in the range [ 1 $-\delta / 2,1+\delta / 2]$. The outer $10 \%$ of the transverse dimensions of the simulational domain are discarded in all plots to avoid possible boundary effects.

The experimental data for the difference between the minimum and the maximum gray scale values for tablets in a given layer (mean tablet widths $\approx 5 \mu \mathrm{m}$ ) in a field of view of $\sim 40 \mu \mathrm{m}$ were compared to the values of the 15 th percentile of the gray scale values in the model at the nucleation sites in each layer.

Acknowledgment. This work was supported by NSF award CHE\&DMR-0613972, DOE award DE-FG02-07ER15899, UW. The Advanced Light Source is supported by the Director, Office of 
Science, Office of Basic Energy Sciences, of the U.S. Department of Energy under Contract No. DE-AC02-05CH11231.

(65) Weiner, S.; Traub, W. FEBS Lett. 1980, 111, 311-316.
(66) Weiner, S.; Traub, W. Phil. Trans. R. Soc. London B 1984, 304, 425434. 